\title{
Evolution of DOHaD: the impact of environmental health sciences
}

\author{
A. C. Haugen, T. T. Schug, G. Collman and J. J. Heindel* \\ Division of Extramural Research and Training, NIEHS, Research Triangle Park, NC, USA
}

\begin{abstract}
Environmental exposures have a significant influence on the chronic health conditions plaguing children and adults. Although the Developmental Origins of Health and Disease $(\mathrm{DOHaD})$ paradigm historically has focused on nutrition, an expanding body of research specifically communicates the effects of chemical exposures on early-life development and the propagation of non-communicable disease across the lifespan. This paper provides an overview of 20 years of research efforts aimed at identifying critical windows of susceptibility to environmental exposures and the signaling changes and epigenetic influences associated with disease progression. DOHaD grants funded by the National Institute of Environmental Health Sciences (NIEHS) in 1991, 2001 and 2011 are identified by grant-analysis software, and each portfolio is analyzed for exposures, disease endpoints, windows of exposure, study design and impact on the field based on publication data. Results show that the 1991 and 2001 portfolios comprised metals, PCBs and air pollutants; however, by 2011, the portfolio has evolved to include or expand the variety of endocrine disruptors, pesticides/persistent organic pollutants and metals. An assortment of brain-health endpoints is most targeted across the portfolios, whereas reproduction and cancer increase steadily over the same time period, and new endpoints like obesity are introduced by 2011 . With mounting evidence connecting early-life exposures to later-life disease, we conclude that it is critical to expand the original DOHaD concept to include environmental chemical exposures, and to continue a research agenda that emphasizes defining sensitive windows of exposure and the mechanisms that cause disease.
\end{abstract}

Received 21 July 2014; Revised 14 October 2014; Accepted 5 November 2014; First published online 4 December 2014

Key words: development, $\mathrm{DOHaD}$, environmental health, exposures, portfolio analysis

\section{Introduction}

Complex diseases result from gene-environment interactions over time; however, historically researchers have relied mostly on genetics for understanding the cause of disease. The dramatic increase of chronic diseases (e.g. diabetes, obesity, some cancers, childhood asthma, attention-deficit hyperactivity disorder, autism, infertility and reproductive dysfunctions such as premature puberty) without concurrent increases in genetic variation does indeed suggest that environmental factors play an important role in disease etiology. In fact, scientists are increasingly examining interactions between genetic background and changes in the environment, such as chemical exposures, diet, stress, infections and drugs.

Early-life environment, beginning at conception, and adult disease was first linked by increased rates of obesity in individuals exposed to famine in utero. ${ }^{1}$ Subsequent studies showed that maternal starvation during the Dutch 'hunger winter' of the Second World War correlated with an increased risk in exposed offspring to cardiovascular disease and metabolic diseases such as obesity, metabolic syndrome and diabetes in adulthood. $^{2}$ In the mid-1980s, David Barker expanded this concept by reporting a negative correlation between birth weight and the rate of death from ischemic heart disease. ${ }^{2,3} \mathrm{He}$

*Address for correspondence: J. J. Heindel, Division of Extramural Research and Training, NIEHS, 530 Davis Drive, RTP, NC 27709, USA.

(Email heindelj@niehs.nih.gov) theorized that normal variations in the transfer of nutrients from mothers to babies have profound long-term implications for the health of offspring and further linked low birth weight, which can indicate poor maternal nutrition, to increased coronary heart disease in adulthood. ${ }^{3}$ These observations paved the way for further research into the link between maternalfetal nutrition and future chronic diseases such as obesity and certain cancers. ${ }^{2,4,5}$ The concept fostered by Dr Barker led to the development of the International Society of Developmental Origins of Health and Disease (DOHaD), which is a registered charity that aims to promote research linking nutrition during pregnancy to disease outcomes across the lifespan. The International Society of $\mathrm{DOHaD}$ also supports a journal by the same name, and an international conference held every other year.

This paper describes the history and evolution of research investigating the role of environmental chemical exposures during development and subsequent disease susceptibility across the lifespan (Fig. 1), as well as provides snapshot portfolio analyses of the National Institute of Environmental Health Sciences (NIEHS)-funded research in 1991, 2001 and 2011 as a surrogate for research in the field. NIEHS is one of the 27 research institutes and centers within the organization, the National Institutes of Health (NIH), and is tasked with the mission to reduce the burden of disease due to environmental influences. Therefore, the institute is a major funder of environmental health research in the United States. To review the 


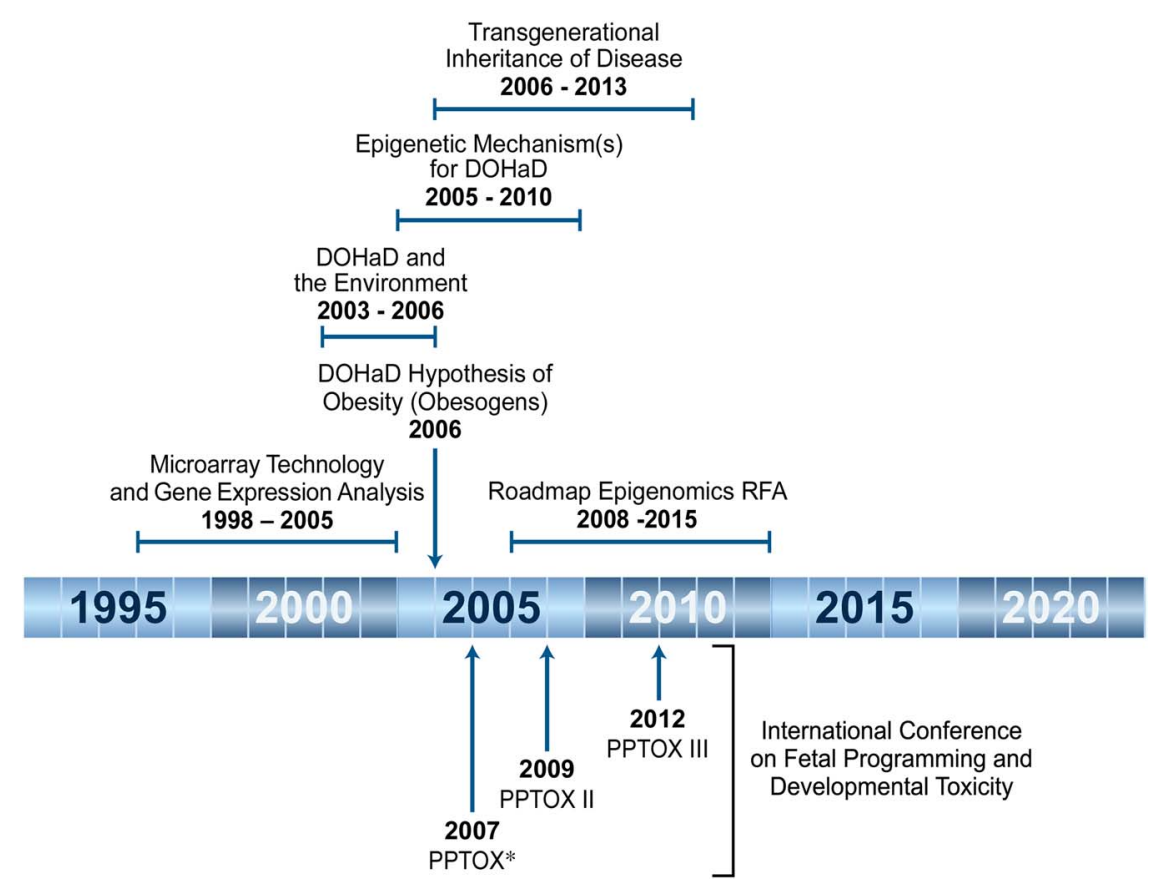

Fig. 1. Science milestones and events that have impacted the field of $\mathrm{DOHaD}$ research as it relates to environmental exposures.

literature and grant data, we chose the definition of $\mathrm{DOHaD}$ that includes early windows of susceptibility (prenatal to early childhood) and increased risk of disease outcome across the lifespan, starting in early childhood. Research grants involving human subjects, model organisms or cell lines are included. Furthermore, the breadth and timing of chemical exposures studied, the disease endpoints measured and the potential mechanisms of action are examined. Finally, we provide suggestions for future $\mathrm{DOHaD}$ research.

\section{The DOHaD paradigm evolves and expands following the Human Genome Project}

While the $\mathrm{DOHaD}$ hypothesis, as it related to humans and nutrition, was developing across Europe, there was another research field that also adapted the $\mathrm{DOHaD}$ hypothesis - toxicology. Early developmental toxicology and environmental health science studies examined chemical exposures primarily during pregnancy, finding that animals exposed to teratogens had altered DNA or mutations that led to death of the fetus, birth defects, low birth weight and premature birth. Alarm over the notion that environmental chemicals could result in disease began to take hold predominantly in the 1970 s over a series of medical tragedies involving diethylstilbestrol (DES). Designed as an artificial estrogen and prescribed from 1940 to 1971 to millions of women during pregnancy to reduce miscarriage, DES was later shown to have serious health consequences (e.g. unusual cancers and reproductive system malformations) for those exposed in utero. Animal studies would later confirm DES's activity as a transplacental carcinogen. ${ }^{6}$ Many of the changes seen in prenatally treated mice were also observed in women exposed to
DES in utero; ${ }^{7}$ in addition to the signal lesion of vaginal clear cell adenocarcinoma, women and mice exposed prenatally to DES also had oviduct malformations, ovarian cysts and histopathological changes in the fallopian tubes. ${ }^{7}$

Although the early teratology literature, including that pertaining to DES, noted that chemicals could also cause 'functional' changes, initially there was no real way to measure them. It was not until the early 1990s that evidence emerged showing how some low-dose chemical exposures without direct teratogenic effects evident at birth could, ultimately, result in later dysfunctions and diseases. ${ }^{8-13}$ These observations were consistent with the emergent $\mathrm{DOHaD}$ concept, shifting the emphasis of developmental toxicology research from the study of mutagens and birth defects to functional changes during development. This change was mostly because of the development of new genomic tools and technologies (e.g. shotgun sequencing, microarray, ChIP-Chip, Agouti mouse model) built on the advances spawned from the NIH-sponsored Human Genome Project. ${ }^{14}$ The genomic revolution helped scientists provide more concrete evidence that early-life exposure to environmental toxicants (e.g. endocrine disruptors) not only cause DNA damage but also can produce more subtle disruptions in the absence of a phenotype at birth, including changes in cell signaling and gene expression, and that such changes are linked to increased susceptibility to disease and dysfunction later in life. ${ }^{15-20}$

\section{Adding more epigenetics research to the $\mathrm{DOHaD}$ portfolio has a lasting impact}

Along with new technologies, such as tissue-specific gene expression profiling, came the examination of epigenetic effects 
(methylation, imprinting and chromatin remodeling) on $\mathrm{DOHaD}$ - an area of research that really began to take off around 2005 (Fig. 1). Epigenetic responses to prenatal exposures were soon linked to health outcomes like cancer, heart and vascular disease, neurodegeneration, asthma, autoimmune disease, male and female reproductive disorders and obesity. The list of environmental chemicals studied also expanded to include xenoestrogens [genistein, (DES), bisphenol A (BPA)], dioxin, organochlorine pesticides, second-hand smoke, phthalates, immunotoxicants and air pollutants. ${ }^{21-26}$ The data from these studies changed the field of environmental health sciences and its thinking about exposures during development and their relationship to epigenetic influences toward diseases later in life. Furthermore, scientists also produced new data on endocrine disruptors and their role in $\mathrm{DOHaD}$ and newly identified health outcomes. For example, in 2006, a hypothesis for the developmental origins of obesity, type 2 diabetes and metabolic syndrome due to endocrine disrupting chemical exposure was published, further stimulating research into the role of such exposures during development (Fig. 1). ${ }^{23,25,27}$

Emerging research on environmental pollutants and epigenetic programming reflected a growing interest by the scientific community, and thus a conference was held in December of 2005 - 'Environmental Epigenomics, Imprinting and Disease Susceptibility. ${ }^{28}$ Some recommendations from the meeting included the following:

- A continued emphasis on fetal or developmental basis of disease paradigm is important with research on both nutrition and environmental exposures and their interactions, as well as epigenetic modification as the mechanism of the increased susceptibility to disease later in life.

- Transgenerational studies (i.e. epigenetic transmission of altered programming across generations) using animal models are needed.

- Epidemiology studies using a case-control design that include epigenetic endpoints are critical to moving the field forward.

- More data on the best way to biobank tissues and serum for future epigenetic analyses are essential to the field.

- Development of new technologies and informatics is needed to properly analyze future epigenetic data.

This conference further stimulated the idea of epigenetic changes as mechanisms of action by environmental agents in the etiology, prevention or progression of disease. Publications over the next several years included examination of epigenetic interference caused by endocrine disruptors leading to breast ${ }^{26}$ and prostate cancer ${ }^{24}$ in animals; role of endocrine disruptors in metastable alleles, imprinting and fetal origins of disease; ${ }^{21}$ and effects of endocrine disruptors on imprinted genes in the mouse embryo. ${ }^{29}$ Another story of success resulting from epigenetic research was data showing that dietary supplements like folic acid or genistein can counteract the hypomethylating effects of BPA exposure during early development, and thus alter the BPA-induced disease trajectory. ${ }^{22}$ Finally, in 2008, the NIH funded the Roadmap Epigenomics Project to expand knowledge in the mechanisms of epigenetic regulation of development, develop new technologies for measuring epigenetic changes, discover new epigenetic marks and to develop and disseminate standardized protocols. This ongoing program has developed complete epigenomes for a variety of cell types and is fostering growth of the field, including assessment of epigenetic marks in human birth cohort studies (www.road mapepigenomics.org; Nature, submitted).

\section{PPTOX conferences highlight progress in $\mathrm{DOHaD}$ and environmental health research}

In order to understand the breadth and depth of new areas of $\mathrm{DOHaD}$ in environmental health research and to bring researchers together to stimulate interactions and collaborations, Philippe Grandjean, who had been examining the effects of Polychlorinated biphenyls (PCBs) on neurodevelopment of children in the Faroe Islands, developed the first international conference on the role of developmental exposures to environmental pollutants and disease outcomes later in life. This conference called Prenatal Programming and Toxicology (PPTOX) was held in the Faroe Islands in 2007 (Fig. 1). The Faroe Statement from PPTOX I ${ }^{30}$ noted that the mother's chemical body burden will be shared with her fetus or neonate, and the child might be exposed to larger doses relative to body weight than adults. In addition, it was stated that susceptibility to a wide range of adverse effects later in life is increased during development (from preconception through adolescence), depending on the organ system and exposures to environmental chemicals. Over the ensuing years, there have been additional PPTOX conferences, with PPTOX II in Miami Beach in 2009 and PPTOX III in Paris in 2012 (Fig. 1). The PPTOX conferences have primarily highlighted progress in the $\mathrm{DOHaD}$ area of environmental chemical exposures, and outcomes from the conferences have been published with several articles appearing in the January 2011 issue of Reproductive Toxicology, ${ }^{31}$ a special-themed issue of the DOHaD Journal (February 2011); Toxicological Sciences; ${ }^{32}$ and Environmental Health. ${ }^{33}$

\section{Analyses of funded DOHaD research in 1991, 2001 and 2011: growth of the field}

A literature review of $\mathrm{DOHaD}$ and environmental chemicals from 1990 to 2013 showed that $50 \%$ of the publications came from NIEHS-funded researchers (data not shown). Thus, here we describe NIEHS funding in the field over a 20-year period. Specifically, a keyword search was used to find active NIEHSfunded grants in the area of $\mathrm{DOHaD}$ for years 1991, 2001 and 2011. Grants meeting the definition of $\mathrm{DOHaD}$ were selected and analyzed using tools within the electronic Scientific Portfolio Assistant (eSPA), which helps build portfolios of NIH research projects for the purpose of analysis and dissemination of information (https://espa.niaid.nih.gov). Search terms used included the following: prenatal, perinatal, postnatal, in utero, 
Table 1. Frequently studied exposures

\begin{tabular}{|c|c|c|c|c|c|}
\hline \multicolumn{2}{|c|}{$1991(n=16)$} & \multicolumn{2}{|c|}{$2001(n=81)$} & \multicolumn{2}{|c|}{$2011(n=173)$} \\
\hline Exposure & $\%$ of total ${ }^{\mathrm{a}}$ & Exposure & $\%$ of total ${ }^{\mathrm{a}}$ & Exposure & $\%$ of total \\
\hline Lead & 50 & Lead & 36 & $\mathrm{BPA}$ & 16 \\
\hline \multirow[t]{10}{*}{ Mercury } & 19 & PCB & 20 & Mercury & 12 \\
\hline & & Mercury & 19 & Organophosphate/chlorine & 12 \\
\hline & & PM/air pollution & 13 & $\mathrm{PCB}$ & 12 \\
\hline & & Organophosphate/chlorine & 10 & PM/air pollution & 10 \\
\hline & & TCDD & 6 & Arsenic & 9 \\
\hline & & & & Lead & 9 \\
\hline & & & & Manganese & 6 \\
\hline & & & & PAH & 6 \\
\hline & & & & PBDE & 6 \\
\hline & & & & Phthalates & 5 \\
\hline
\end{tabular}

BPA, bisphenol A; PM, particulate matter; TCDD, 2,3,7,8-tetrachlorodibenzo-p-dioxin; PCB, polychlorinated biphenyl; PAH, polycyclic aromatic hydrocarbons; PBDE, polybrominated diphenyl ethers.

${ }^{a}$ Any exposures accounting for $<5 \%$ of the portfolio are not included.

Table 2. Frequently studied diseaselorgan endpoints

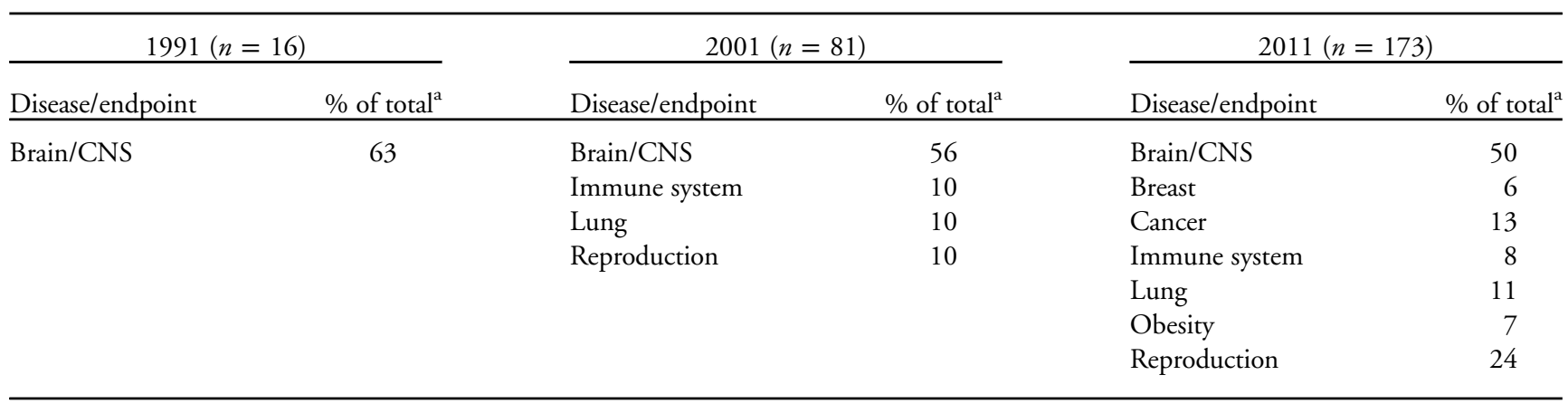

CNS, central nervous system.

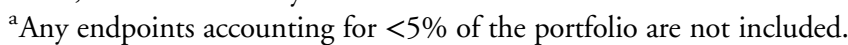

fetus, pregnant (cy), child (ren), early childhood, puberty and development (al). We also chose to analyze only research grant mechanisms and treated larger multicomponent grants, such as the Children's Environmental Health Centers, as single projects. Finally, the final portfolios and analyses were reviewed by and discussed within a working group (see acknowledgment section). Each portfolio was then analyzed for the following: program mechanisms; scientific mechanisms; toxicity endpoints; disease/organ endpoints; human subjects, model organisms, or both; windows of susceptibility; and productivity based on publication numbers, citations and journal impact factors. Publication data were attained through eSPA (http://connection. ebscohost.com/c/articles/79702830/electronic-scientific-portfolioassistant-integrating-scientific-knowledge-databases-supportprogram-impact-assessment) and SPIRES. The portfolios were further analyzed using the Information for Management, Planning, Analysis and Coordination II data system (www.tfgov. com/data/NIH.gov/Pages/era.nih.gov\%5Dimpacii\%5Dindex.cfm) to determine funding history and total dollars spent.

\section{Early-life exposures to lead and mercury are a priority in the 1991 portfolio}

In 1991, the NIEHS DOHaD portfolio contained 16 grants. Metals were the most studied environmental exposures during this period, with lead and mercury accounting for $69 \%$ of the $\mathrm{DOHaD}$ grants (Table 1), and the brain and/or central nervous system (CNS; e.g. neurodevelopment, neurocognition, neurobehavior, neuropsychiatry and neurobiology) were the most studied organ systems (Table 2). Most of the studies documented a robust relationship between in utero and postnatal exposure to lead and the long-term neuropsychological morbidities, as well as resulting juvenile delinquency and criminality during early adulthood. ${ }^{13,34-42}$ As a consequence of these and other studies, the Centers for Disease Control and Prevention lowered the lead action level for children to $10 \mu \mathrm{g} / \mathrm{dl}$, and more recently set a 'reference value' of $5 \mu \mathrm{g} / \mathrm{dl}$ (see http://www.cdc.gov/nceh/lead/ACCLPP/blood_lead_levels. $\mathrm{htm})$. Research on methylmercury exposure in the Faroe 
(a)

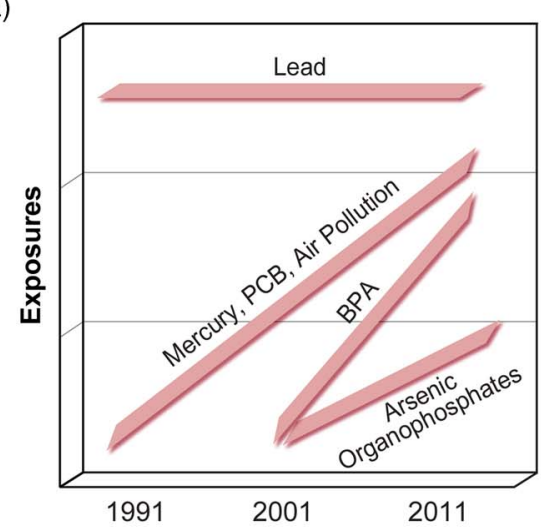

(c)

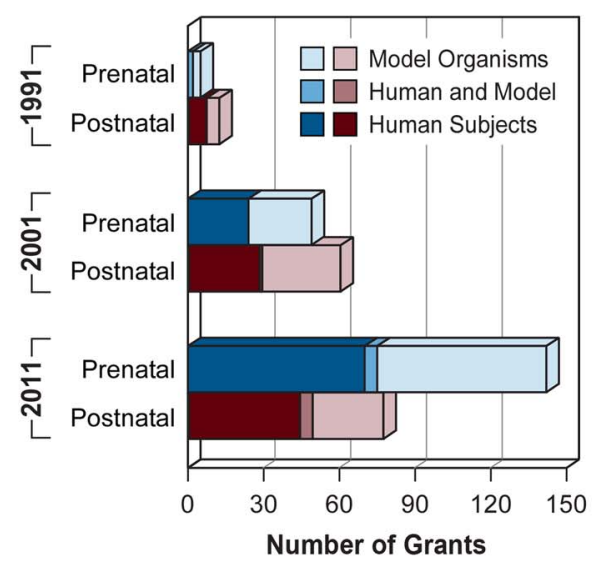

(b)

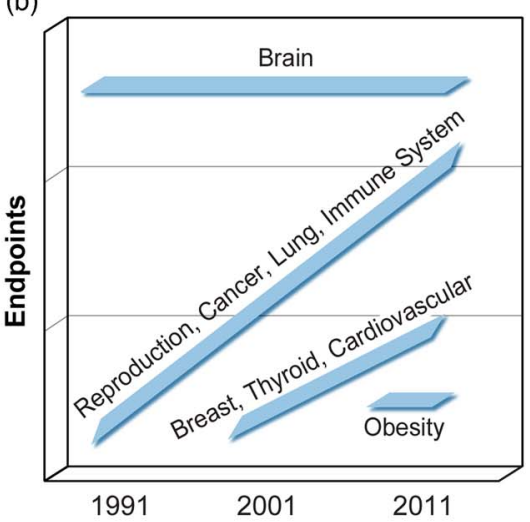

(d) Dollar Amount of Grants (0, Millions of Dollars)

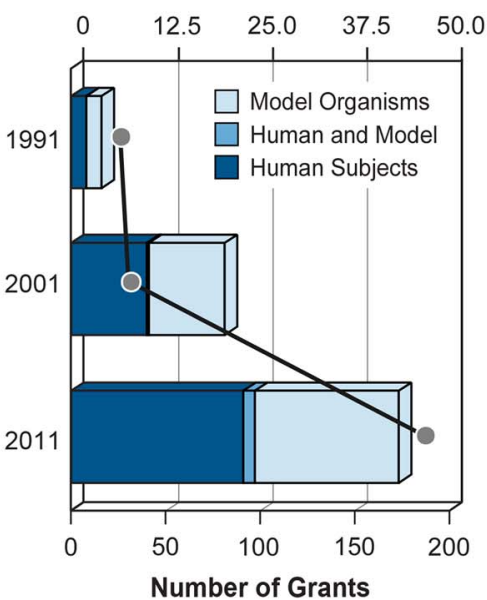

Fig. 2. (a) Overview of the timing of funded disease/organ endpoint studies and relative predominance in each portfolio (1991, 2001 and 2011); (b) overview of the timing of funded toxicity endpoint studies and relative predominance in each portfolio; (c) content analysis of three grant portfolios (1991, 2001 and 2011) for windows of exposure and human subject studies $v$. model organisms, or both (note: if a grant assessed more than one endpoint, each was counted individually); (d) content analysis of three grant portfolios (1991, 2001 and 2011) - the number of grants; human subject studies $v$. model organisms or both (note: if a grant assessed more than one endpoint, each was counted individually); and total dollars spent for each portfolio.

Islands, where marine animals and fish are food staples, also revealed its damaging effects on neurodevelopment and subsequent neuropsychological deficits later in life. ${ }^{43-47}$ As a result of these and other studies, the EPA, National Research Council, World Health Organization and United Nations Environment Program supported the established reference dose of $0.1 \mu \mathrm{g} / \mathrm{kg} / \mathrm{day}$ for a safe exposure limit for the mother and fetus. ${ }^{37,48-51}$ Finally, although all 16 projects fit the definition of $\mathrm{DOHaD}$, only one project included the discussion of regulated gene expression and developmental programming within the specific aims of the grant. ${ }^{52}$

\section{The 2001 portfolio points to evolving DOHaD research, including developmental programming}

The NIEHS 2001 portfolio grew to 81 active research projects fitting the $\mathrm{DOHaD}$ paradigm. The portfolio again centered mainly on metals, with lead and mercury accounting for $55 \%$ of the entire group (Table 1; Fig. 2a). It was more robust and diverse than the 1991 portfolio and included $\mathrm{DOHaD}$ research on developmental exposures to PCBs, air pollution, organophosphates and organochlorines (Table 1 and Fig. 2a). NIEHS was also funding $\mathrm{DOHaD}$ grants researching reproductive, lung and immune system diseases $(10 \%$ of total; Table 2 and Fig. 2b). The majority of grants still centered on postnatal or early childhood exposures rather than prenatal exposures, with the numbers of animal and human studies remaining roughly equal (Fig. 2c). Moreover, during this time, a small number of the grants began exploring some form of developmental programming.

\section{The 2011 NIEHS DOHaD portfolio includes more exposures, mechanisms and health outcomes}

Between 2001 and 2011, the literature on $\mathrm{DOHaD}$ and environmental chemical exposures underwent a dramatic 


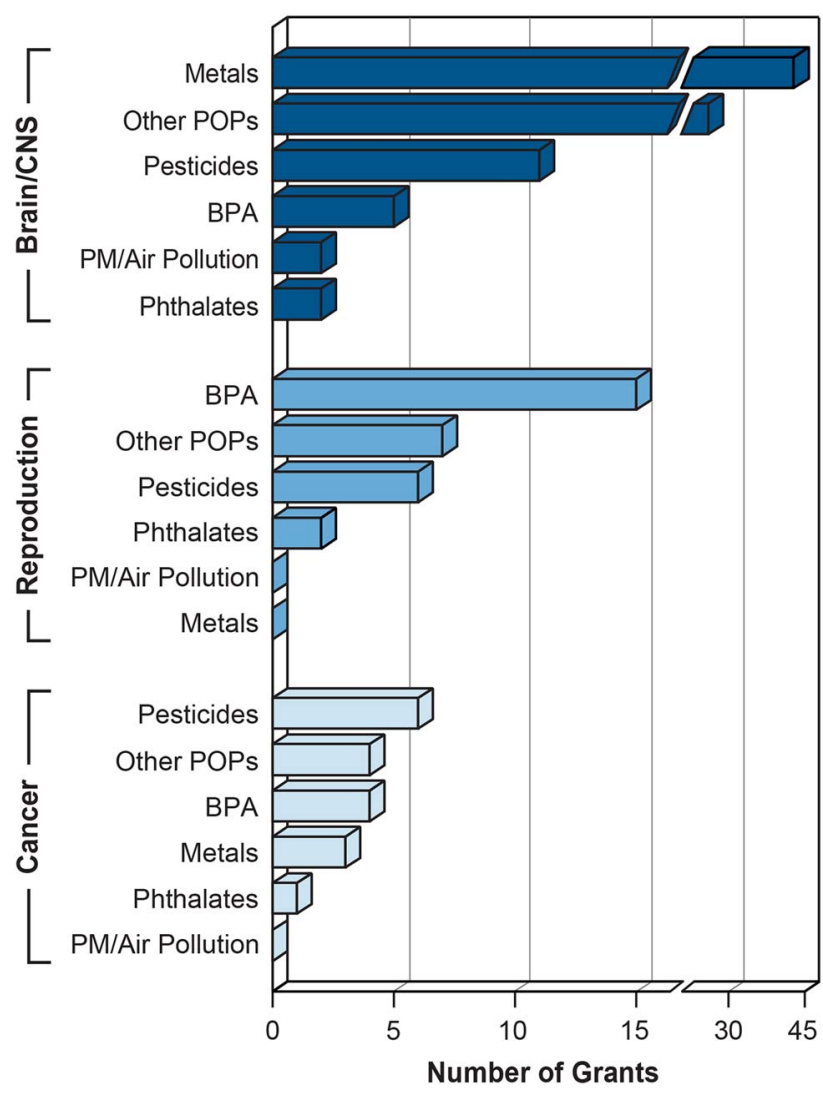

Fig. 3. Most studied disease/organ endpoints and associated toxicity endpoints.

increase. The NIEHS DOHaD portfolio mirrored this change as the number of grants increased to 173 by 2011 . The snapshot analysis of the 2011 portfolio of active grants revealed that the number of grant investments in studying model organisms $v$. human subjects remained about equal as previous years (Fig. 2d). (Note: the vast majority of model organism studies involved mice and rats.) However, the number of grants describing pesticides and other endocrine disruptors (e.g. BPA) peaked in the $2011 \mathrm{DOHaD}$ portfolio (Table 1 and Fig. 2a), and the number and types of persistent organic pollutants (POPs) and metals also expanded. Grants centered on reproductive diseases and disorders, including sex differences, increased from $10 \%$ in 2001 to $24 \%$ in 2011 , and studies involving the brain/CNS, cancer and breast at least doubled in number during that same time period (Table 2 and Fig. 2b). In addition, endpoints such as obesity $(7 \%)$ were observed for the first time in the 2011 portfolio (Table 2 and Fig. 2b). The most studied disease or health endpoints (brain/CNS, reproduction and cancer) were analyzed for concomitant exposures (Fig. 3), and the largest number of brain/CNS grants were associated with metal exposures (e.g. arsenic, lead, mercury and manganese), with many others describing pesticides (e.g. organophosphates and organochlorines) and other POPs (e.g. polybrominated biphenyl ethers (PBDEs), polychlorinated biphenyls (PCBs), and polycyclic aromatic hydrocarbons (PAHs); Fig. 3). On the other hand, grants
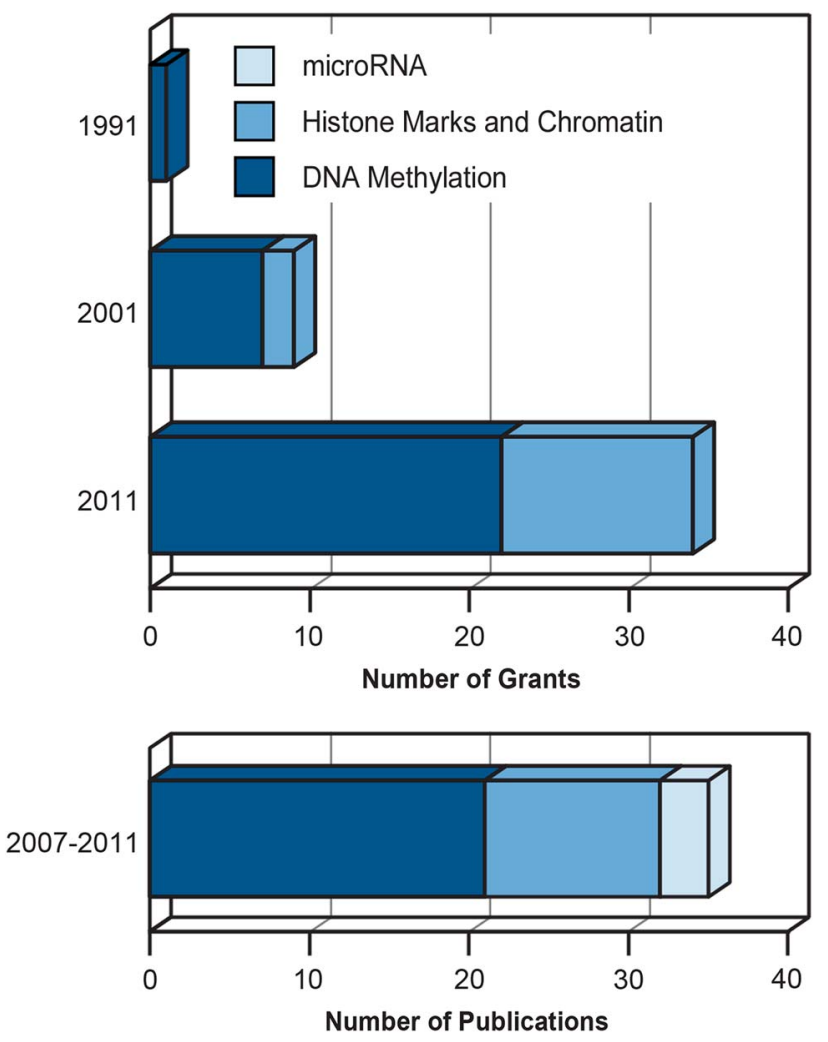

Fig. 4. (a) Epigenetics-based $\mathrm{DOHaD}$ grants across three grant portfolios (1991, 2001 and 2011) (note: if a grant studied more than one epigenetic process, each was counted individually). Also shown, the evolution of epigenetics research from examining mostly DNA methylation to including chromatin remodeling and regulatory RNAs; (b) The number of publications related to NIEHS $\mathrm{DOHaD}$ grants with an epigenetic focus during the years from 2007 to 2011. NIEHS, National Institute of Environmental Health Sciences; DOHaD, Developmental Origins of Health and Disease.

involving reproductive dysfunctions in males and/or females were primarily affiliated with BPA exposure followed by pesticides and other POPs (Fig. 3).

By 2011, the number of studies on prenatal exposures nearly doubled with 142 grants as compared with 77 postnatal exposure grants (Fig. 2c). This increase in prenatal exposure studies follows an emerging trend toward identifying epigenetic mechanisms (e.g. DNA methylation, histone modifications, chromatin remodeling and RNA regulation/metabolism). In fact, 35 of the 173 grants in the $\mathrm{DOHaD}$ portfolio specifically explored epigenetic changes. As a comparison, there was just one epigenetics-related grant in 1991 and 11 grants in 2001 (Fig. 4a). Not only did the number of epigenetic grants within $\mathrm{DOHaD}$ increase, but the epigenetics research itself evolved from analyzing only DNA methylation to including chromatin remodeling.

Having observed this increasing trend in epigenetics research, we analyzed publications resulting from the NIEHSfunded $\mathrm{DOHaD}$ projects, as changes in science are reflected more quickly in publications rather than in grant applications. 
Table 3. Journals publishing most cited articles

\begin{tabular}{llcc}
\hline DOHaD portfolio & Journal & \% of top 20 articles cited & Frequent exposures \\
\hline 1991 & Toxicology and Applied Pharmacology & $30 \%$ & Dioxin \\
& Neurotoxicity and Teratology & $15 \%$ & Lead \\
& Environmental Health Perspectives & $15 \%$ & Mercury \\
2011 & Environmental Health Perspectives & $40 \%$ & BPA \\
& Proceedings of the National Academy of Sciences & $10 \%$ & PCBs \\
& Lancet & $10 \%$ & Organophosphates
\end{tabular}

Interestingly, epigenetics-related publications (2007-2011) showed a clear uptick in discussions regarding histone marks, chromatin modifications and regulatory RNAs (Fig. 4b). This result follows a trend in the epigenetics field of the progression of studying primarily DNA methylation to identifying modifications in RNA metabolism and chromatin structure. Indeed, new discoveries and technologies are allowing scientists to explore the genome in much greater detail to determine epigenetic susceptibility to environmental exposures. Some grants in the 2011 portfolio also began research into transgenerational inheritance - environmentally induced changes in the epigenome that are inherited due to incomplete germline reprogramming. ${ }^{53-56}$ For example, Anway et al. ${ }^{54,57}$ reported transgenerational effects of reproductive toxicity resulting in decreased sperm count and motility in male rats following maternal exposure to the fungicide, vinclozolin and the pesticide methoxychlor. Surprisingly, the effects on fertility were observed in four subsequent generations. ${ }^{57}$ We expect more studies to broaden the characterization of epigenetic mechanisms to more than only DNA methylation, as well as produce data that demonstrates epigenetic changes as predictive biomarkers of disease susceptibility.

\section{Publication data reflect the evolution and impact of NIEHS- funded $\mathrm{DOHaD}$ research}

In order to assess whether the types of journals that published DOHaD-related research changed over time, the top publications, based on citation number, were researched for both the 1991 and 2011 grant portfolios. The 2001 portfolio was not included because of overlap in the grants of both the 1991 and 2011 portfolios, thereby causing publication redundancy. The journals that published the most cited articles resulting from grants in the 1991 portfolio mirrored the research trends of that time and were Toxicology and Applied Pharmacology (6 of 20), Neurotoxicology and Teratology (3 of 20) and Environmental Health Perspectives (EHP; 3 of 20) (Table 3 and Supplementary Table S1). The journals that published the most cited articles resulting from grants in the 2011 portfolio were Lancet (2 of 20), Proceedings of the National Academy of Sciences (PNAS) (2 of 20) and EHP (8 of 20) (Table 3 and Supplementary Table S2). This change in journals follows the evolving science from general toxicity and birth defects to chemicals such as endocrine disruptors and functional changes that lead to susceptibility to non-communicable diseases across the lifespan. In 1991, the most cited articles discussed dioxin, lead and mercury exposures (Table 1 and Supplementary Table S1), whereas in 2011 the most cited articles reflected an expanded number of chemicals including BPA, PCBs and pesticides like organophosphates (Table 1 and Supplementary Table S2). Citation numbers for the entire 2011 portfolio, including both research and review articles, added up to over 17,000 (data not shown). It is clear from these data that research across the globe and international scientific conferences have all worked together to expand research areas, fill identified gaps, enhance our knowledge of the effects of developmental exposures and broaden our reach in the form of high-impact publications.

\section{Data gaps}

\section{New trajectories to stimulate the $\mathrm{DOHaD}$ field}

As noted, studies on the effects of metal exposures (primarily lead and mercury) on neurodevelopment have consistently occupied a large percentage of the $\mathrm{DOHaD}$ portfolio at NIEHS (Fig. 2a and Table 1); however, the last decade also saw an increase in environmental health researchers studying the effects of endocrine-disrupting chemicals such as BPA, phthalates, tributyl tin, PCBs, flame retardants, pesticides, dioxins and other persistent organic pollutants. In fact, the increase in grants with reproductive endpoints reflects the growing interest within the $\mathrm{DOHaD}$ research community on endocrine disruptors and downstream differential sex effects. The NIEHS, for its part, has also highlighted both epigenetics and $\mathrm{DOHaD}$ as high priority areas for the next several years in its newly released Strategic Plan. ${ }^{58}$ Moreover, emerging $\mathrm{DOHaD}$ research areas include new disease/dysfunction endpoints such as obesity, diabetes and metabolic syndrome, as well as diseases of other endocrine systems that might be affected by environmental exposures.

It is imperative that scientists continue to work on better understanding how exposure affects the regulatory proteins and processes upstream of DNA methylation and other epigenetic marks. Moving the field from descriptive and correlative studies to an enhanced mechanistic understanding of how environmental exposures affect the proteins and functional genomic 
elements involved in establishing and maintaining gene expression patterns and chromatin states is vital. As there are now studies showing multigenerational and transgenerational inheritance of disease/dysfunctions, understanding the mechanism(s) for transgenerational inheritance as well as which chemicals can transmit deleterious effects to subsequent generations and targeted tissues are important goals within the $\mathrm{DOHaD}$ field. In addition, the sensitivity of the F2 generation (e.g. grandchildren) from exposure during pregnancy has not been studied well. The F1 generation (direct offspring) is somatically exposed during development, but the F2 generation is exposed only via the germ cells; therefore, transmission to the F2 generation could be somewhat different and significant. Indeed, there are now numerous publications showing that developmental exposures to environmental chemicals including DDT, tributyl tin, dioxin, vinclozolin and phthalates can lead to effects that are apparent in the F3 and F4 generations. ${ }^{25,56,59-64}$

Although the $\mathrm{DOHaD}$ paradigm centers on the in utero and/ or neonatal time period as a sensitive window for environmental exposures, it is important to define all sensitive windows of exposure across the lifespan, and to understand what underlies a 'sensitive window.' It is also critical to better mimic real world situations, including determining the interaction of multiple exposures (mixtures) or chemicals, and indeed mixtures of environmental exposures with altered nutrition, stress, infection and/or drugs. Similarly, expanding $\mathrm{DOHaD}$ experiments beyond the analysis of one disease is important, in other words, looking for disease syndromes, as an environmental chemical is likely to affect more than one organ/tissue, and thus potentially increasing the risk for many diseases. If researchers continue to study the one-chemical and one-disease relationship, it will be very difficult to completely understand the integrated toxicity associated with exposures.

Epigenetic signatures or footprints that result from developmental exposures, which can be predictive of increased disease risk later in life, must be developed. Specifically, signatures from accessible tissues like cord blood/tissue and blood cells would be particularly valuable. Although researchers are currently linking specific epigenetic signatures in blood to disease outcomes in humans a few years later, these signatures have not been validated with regard to sensitivity, specificity or accuracy. Does an epigenetic footprint in blood really predict disease in a tissue? Once it is possible to predict lifetime disease incidence due to developmental exposures, effective strategies can be devised to reduce exposure to stressors in order to reduce disease incidence. Such strategies have the potential to reduce the overall societal burden of non-communicable disease and alleviate health disparities. ${ }^{32}$

Finally, as noted in the published summary of the PPTOX III meeting, ${ }^{32}$ the two fields of research, developmental nutrition (i.e. the original $\mathrm{DOHaD}$ focus) and developmental environmental/chemical exposures, need to work together as many aspects of the research are similar. For example, both act during specific windows of exposure when tissues are developing; both result in functional changes that can be apparent without a change in birth weight and appear as increased disease susceptibility after a latent period; both alter developmental 'programming,' which at least in part is due to altered epigenetic marks and effects of both nutrition and chemical exposures that can be transmitted to future generations via the germ line; and both result in increased susceptibility to major human diseases including obesity, diabetes, cardiovascular diseases and neurodevelopmental and reproductive diseases. Thus, as noted, research on nutrition and environmental chemicals in $\mathrm{DOHaD}$ can be viewed as two sides of the same coin. ${ }^{32}$ To truly improve human health by reducing the burden of non-communicable diseases, scientists must explore both areas with the goal of improving nutrition and reducing environmental chemical exposures during development across the globe.

\section{Conclusions}

Non-communicable diseases and conditions result from a combination of genetic and environmental factors, and the most sensitive window for environmental impact is during development. Data described in the body of the literature regarding nutrition and $\mathrm{DOHaD}$ led to the development of the ' $\mathrm{DOHaD}$ paradigm.' It is now clear that a wide variety of environmental exposures occurring during periods of time when tissues and organ systems are developing can also markedly increase the risk for (or even cause) disease across the life course. ${ }^{32}$ Indeed, research on environmental chemicals has expanded the $\mathrm{DOHaD}$ paradigm to include more diseases/dysfunctions; a variety of environmental exposures, singly and in combination; and also epigenetic mechanisms, which include transmission of the disease/dysfunction susceptibility across multiple generations. Evidence also suggests that disadvantaged populations may experience greater exposure to environmental hazards and exhibit higher rates of disease incidence, morbidity and mortality. Understanding and modulating this risk in humans during sensitive windows of development offers the promise of disease prevention and reduction of health disparities. ${ }^{32}$

\section{Acknowledgments}

The authors thank the NIEHS Division of Extramural Research and Training Program administrators involved in $\mathrm{DOHaD}$, endocrine disruptors and epigenetics for their guidance in developing and reviewing the manuscript: Cindy Lawler, Lisa Chadwick, Carolyn Dilworth, Kimberly Gray, Michael Humble, Annette Kirshner, Sri Nadadur, Les Reinlib and Fred Tyson. In addition, the authors thank Michael Loewe for help with financial data, Sue Edelstein for graphics and Heather King and Marisa Naujokas for copyediting and reviewing the manuscript.

\section{Financial Support}

This research received no specific grant from any funding agency, commercial or not-for-profit sectors. 


\section{Conflicts of Interest}

None.

\section{Supplementary material}

To view supplementary material for this article, please visit http://dx.doi.org/10.1017/S2040174414000580

\section{References}

1. Ravelli GP, Stein ZA, Susser MW. Obesity in young men after famine exposure in utero and early infancy. N Engl J Med. 1976; 295, 349-353.

2. Barker DJ, Osmond C. Infant mortality, childhood nutrition, and ischaemic heart disease in England and Wales. Lancet. 1986; 1, 1077-1081.

3. Barker DJ, Winter PD, Osmond C, Margetts B, Simmonds SJ. Weight in infancy and death from ischaemic heart disease. Lancet. 1989; 2, 577-580.

4. Barker DJ. Deprivation in infancy and risk of ischaemic heart disease. Lancet. 1991; 337, 981.

5. Barker DJ, Gluckman PD, Godfrey KM, et al. Fetal nutrition and cardiovascular disease in adult life. Lancet. 1993; 341, 938-941.

6. McLachlan JA, Newbold RR, Bullock BC. Long-term effects on the female mouse genital tract associated with prenatal exposure to diethylstilbestrol. Cancer Res. 1980; 40, 3988-3999.

7. Newbold RR, Bullock BC, McLachlan JA. Progressive proliferative changes in the oviduct of mice following developmental exposure to diethylstilbestrol. Teratog Carcinog Mutagen. 1985; 5, 473-480.

8. Burbacher TM, Rodier PM, Weiss B. Methylmercury developmental neurotoxicity: a comparison of effects in humans and animals. Neurotoxicol Teratol. 1990; 12, 191-202.

9. Gilbert SG, Grant-Webster KS. Neurobehavioral effects of developmental methylmercury exposure. Environ Health Perspect. 1995; 103(Suppl. 6), 135-142.

10. Bellinger D, Sloman J, Leviton A, et al. Low-level lead exposure and children's cognitive function in the preschool years.

Pediatrics. 1991; 87, 219-227.

11. Bellinger D, Dietrich KN. Low-level lead exposure and cognitive function in children. Pediatr Ann. 1994; 23, 600-605.

12. Banks EC, Ferretti LE, Shucard DW. Effects of low level lead exposure on cognitive function in children: a review of behavioral, neuropsychological and biological evidence. Neurotoxicology. 1997; 18, 237-281.

13. Needleman HL, Schell A, Bellinger D, Leviton A, Allred EN. The long-term effects of exposure to low doses of lead in childhood. An 11-year follow-up report. N Engl J Med. 1990; 322, 83-88.

14. Venter JC, Adams MD, Myers EW, et al. The sequence of the human genome. Science. 2001; 291, 1304-1351.

15. Wadhwa PD, Buss C, Entringer S, Swanson JM. Developmental origins of health and disease: brief history of the approach and current focus on epigenetic mechanisms. Semin Reprod Med. 2009; 27, 358-368.

16. Callinan PA, Feinberg AP. The emerging science of epigenomics. Hum Mol Genet. 2006; 15(Spec No. 1), R95-R101.

17. Huang WW, Yin Y, Bi Q, et al. Developmental diethylstilbestrol exposure alters genetic pathways of uterine cytodifferentiation. Mol Endocrinol. 2005; 19, 669-682.
18. Lehmann KP, Phillips S, Sar M, Foster PM, Gaido KW. Dose-dependent alterations in gene expression and testosterone synthesis in the fetal testes of male rats exposed to di (n-butyl) phthalate. Toxicol Sci. 2004; 81, 60-68.

19. Ruden DM, Xiao L, Garfinkel MD, Lu X. Hsp90 and environmental impacts on epigenetic states: a model for the transgenerational effects of diethylstibesterol on uterine development and cancer. Hum Mol Genet. 2005; 14(Spec No. 1), R149-R155.

20. Bhattacherjee V, Mukhopadhyay P, Singh S, et al. Neural crest and mesoderm lineage-dependent gene expression in orofacial development. Differentiation. 2007; 75, 463-477.

21. Dolinoy DC, Weidman JR, Jirtle RL. Epigenetic gene regulation: linking early developmental environment to adult disease. Reprod Toxicol. 2007; 23, 297-307.

22. Dolinoy DC, Huang D, Jirtle RL. Maternal nutrient supplementation counteracts bisphenol A-induced DNA hypomethylation in early development. Proc Natl Acad Sci US A. 2007; 104, 13056-13061.

23. Grun F, Blumberg B. Environmental obesogens: organotins and endocrine disruption via nuclear receptor signaling. Endocrinology. 2006; 147(Suppl.), S50-S55.

24. Keri RA, Ho SM, Hunt PA, et al. An evaluation of evidence for the carcinogenic activity of bisphenol A. Reprod Toxicol. 2007; 24, $240-252$.

25. Schug TT, Janesick A, Blumberg B, Heindel JJ. Endocrine disrupting chemicals and disease susceptibility. J Steroid Biochem Mol Biol. 2011; 127, 204-215.

26. Soto AM, Maffini MV, Sonnenschein C. Neoplasia as development gone awry: the role of endocrine disruptors. Int $\mathrm{J}$ Androl. 2008; 31, 288-293.

27. Janesick A, Blumberg B. Obesogens, stem cells and the developmental programming of obesity. Int J Androl. 2012; 35, 437-448.

28. Heindel JJ, McAllister KA, Worth L Jr, Tyson FL. Environmental epigenomics, imprinting and disease susceptibility. Epigenetics. 2006; 1, 1-6.

29. Kang ER, Iqbal K, Tran DA, et al. Effects of endocrine disruptors on imprinted gene expression in the mouse embryo. Epigenetics. 2011; 6, 937-950.

30. Grandjean P, Weihe P. Developmental origins of environmentally induced disease and dysfunction International Conference on Foetal Programming and Developmental Toxicity, Torshavn, Faroe Islands, 20-24 May, 2007. Basic Clin Pharmacol Toxicol. 2008; 102, 71-72.

31. Darney S, Fowler B, Grandjean P, et al. Prenatal Programming and Toxicity II (PPTOX II): role of environmental stressors in the developmental origins of disease. Reprod Toxicol. 2011; 31, 271.

32. Schug TT, Barouki R, Gluckman P, et al. PPTOX III: environmental stressors in the developmental origins of disease: evidence and mechanisms. Toxicol Sci. 2013; 131, 343-350.

33. Barouki R, Gluckman PD, Grandjean P, Hanson M, Heindel JJ. Developmental origins of non-communicable disease: implications for research and public health. Environ Health. 2012; 11, 42 (http://www.ehjournal.net/content/11/1/42).

34. Needleman HL, McFarland C, Ness RB, Fienberg SE, Tobin MJ. Bone lead levels in adjudicated delinquents. A case control study. Neurotoxicol Teratol. 2002; 24, 711-717.

35. Needleman HL, Riess JA, Tobin MJ, Biesecker GE, Greenhouse JB. Bone lead levels and delinquent behavior. JAMA. 1996; 275, 363-369. 
36. Dietrich KN, Succop PA, Berger OG, Hammond PB, Bornschein RL. Lead exposure and the cognitive development of urban preschool children: the Cincinnati Lead Study cohort at age 4 years. Neurotoxicol Teratol. 1991; 13, 203-211.

37. Lanphear BP, Hornung R, Khoury J, et al. Low-level environmental lead exposure and children's intellectual function: an international pooled analysis. Environ Health Perspect. 2005; 113, 894-899.

38. Pounds JG, Long GJ, Rosen JF. Cellular and molecular toxicity of lead in bone. Environ Health Perspect. 1991; 91, 17-32.

39. Rice DC, Evangelista de Duffard AM, Duffard R, et al. Lessons for neurotoxicology from selected model compounds: SGOMSEC joint report. Environ Health Perspect. 1996; 104 (Suppl. 2), 205-215.

40. Torres-Sanchez LE, Berkowitz G, Lopez-Carrillo L, et al. Intrauterine lead exposure and preterm birth. Environ Res. 1999; 81, 297-301.

41. Haynes EN, Chen A, Ryan P, et al. Exposure to airborne metals and particulate matter and risk for youth adjudicated for criminal activity. Environ Res. 2011; 111, 1243-1248.

42. Grandjean P, White RF. Neurobehavioral dysfunction as a possible sentinel of methylmercury exposure. Hum Ecol Risk Assess. 2001; 7, 1079-1089.

43. Grandjean P, Weihe P, Needham LL, et al. Relation of a seafood diet to mercury, selenium, arsenic, and polychlorinated biphenyl and other organochlorine concentrations in human milk. Environ Res. 1995; 71, 29-38.

44. Dalgard C, Grandjean P, Jorgensen PJ, Weihe P. Mercury in the umbilical cord: implications for risk assessment for minamata disease. Environ Health Perspect. 1994; 102, 548-550.

45. Grandjean P, Weihe P. Neurobehavioral effects of intrauterine mercury exposure: potential sources of bias. Environ Res. 1993; 61, 176-183.

46. Grandjean P, Weihe P, Jorgensen PJ, et al. Impact of maternal seafood diet on fetal exposure to mercury, selenium, and lead. Arch Environ Health. 1992; 47, 185-195.

47. Grandjean P, Weihe P, White RF, et al. Cognitive deficit in 7-year-old children with prenatal exposure to methylmercury. Neurotoxicol Teratol. 1997; 19, 417-428.

48. Rice DC. The US EPA reference dose for methylmercury: sources of uncertainty. Environ Res. 2004; 95, 406-413.

49. Murata K, Budtz-Jorgensen E, Grandjean P. Benchmark dose calculations for methylmercury-associated delays on evoked potential latencies in two cohorts of children. Risk Anal. 2002; 22, 465-474.
50. Budtz-Jorgensen E, Grandjean P, Keiding N, White RF, Weihe P. Benchmark dose calculations of methylmercuryassociated neurobehavioural deficits. Toxicol Lett. 2000; 112-113, 193-199.

51. Budtz-Jorgensen E, Grandjean P, Weihe P. Separation of risks and benefits of seafood intake. Environ Health Perspect. 2007; 115, 323-327.

52. McGivern RF, Sokol RZ, Berman NG. Prenatal lead exposure in the rat during the third week of gestation: long-term behavioral, physiological, and anatomical effects associated with reproduction. Toxicol Appl Pharmacol. 1991; 110, 206-215.

53. Crews D, Gore AC, Hsu TS, et al. Transgenerational epigenetic imprints on mate preference. Proc Natl Acad Sci U S A. 2007; 104, 5942-5946.

54. Anway MD, Rekow SS, Skinner MK. Transgenerational epigenetic programming of the embryonic testis transcriptome. Genomics. 2008; 91, 30-40.

55. Guerrero-Bosagna C, Settles M, Lucker B, Skinner MK. Epigenetic transgenerational actions of vinclozolin on promoter regions of the sperm epigenome. PLoS One. 2010; 5, e13100.

56. Skinner MK, Manikkam M, Guerrero-Bosagna C. Epigenetic transgenerational actions of environmental factors in disease etiology. Trends Endocrinol Metab. 2010; 21, 214-222.

57. Anway MD, Cupp AS, Uzumcu M, Skinner MK. Epigenetic transgenerational actions of endocrine disruptors and male fertility. Science. 2005; 308, 1466-1469.

58. Birnbaum LS. NIEHS's new strategic plan. Environ Health Perspect. 2012; 120, a298.

59. Rutter M, Jones RR (eds). Lead versus health: sources and effects of low level lead exposure. Arch Dis Child. 1983; 58, 759.

60. Skinner MK, Anway MD, Savenkova MI, Gore AC, Crews D. Transgenerational epigenetic programming of the brain transcriptome and anxiety behavior. PLoS One. 2008; 3, e3745.

61. Skinner MK, Manikkam M, Guerrero-Bosagna C. Epigenetic transgenerational actions of endocrine disruptors. Reprod Toxicol. 2011; 31, 337-343.

62. Wolstenholme JT, Edwards M, Shetty SR, et al. Gestational exposure to bisphenol a produces transgenerational changes in behaviors and gene expression. Endocrinology. 2012; 153, 3828-3838.

63. Wolstenholme JT, Taylor JA, Shetty SR, et al. Gestational exposure to low dose bisphenol A alters social behavior in juvenile mice. PLoS One. 2011; 6, e25448.

64. Wolstenholme JT, Rissman EF, Connelly JJ. The role of bisphenol A in shaping the brain, epigenome and behavior. Horm Behav. 2011; 59, 296-305. 